SLAC-TN-10-055

LCLS-TN-06-17

\title{
LCLS Undulator Test Plan
}

\author{
Zachary Wolf ${ }^{a}$, Yurii Levashov ${ }^{a}$, Heinz-Dieter Nuhn $^{a}$, Isaac Vasserman ${ }^{b}$ \\ ${ }^{a}$ Stanford Linear Accelerator Center \\ ${ }^{b}$ Argonne National Laboratory
}

December 11, 2006

\begin{abstract}
This note presents the test plan for the LCLS undulators. The undulators will be measured and tuned in the Magnetic Measurement Facility at SLAC. The requirements for tuning are well established and are summarized. A brief discussion of the measurement equipment is presented. This is followed by the detailed test plan in which each step is enumerated. Finally, the measurement results and storage format are presented.
\end{abstract}

\section{$1 \quad$ Introduction $^{1}$}

The LCLS consists of 33 undulator segments, hereafter referred to as undulators, plus 6 spares and one reference undulator. The undulators must be tuned to meet strict requirements. They must also be fiducialized to allow alignment with other components. This note details the plan for tuning and fiducializing the LCLS undulators.

The note begins with the list of tuning and fiducialization requirements. The laboratory in which the work will be performed and the relevant equipment is then briefly described. This is followed by a detailed test plan in which all the steps of tuning and fiducialization are enumerated.

\section{Requirements}

The LCLS undulator tuning and fiducialization requirements come from an undulator Physics Requirements Document ${ }^{2}$. The list of tuning and fiducialization requirements are briefly summarized below. These requirements must be met for all horizontal undulator positions within $\pm 2.5 \mathrm{~mm}$ of the undulator beam axis and all vertical positions within $\pm 200 \mu \mathrm{m}$ of the beam axis.

1. The undulator temperature at which all measurements are performed must be $20.0 \pm$ 0.1 degrees Celsius. The Hall probe temperature at which all measurements are performed must be $20.0 \pm 0.3$ degrees Celsius.

\footnotetext{
${ }^{1}$ Work supported in part by the DOE Contract DE-AC02-76SF00515. This work was performed in support of the LCLS project at SLAC.

${ }^{2}$ H. D. Nuhn et al., "General Undulator System Requirements", LCLS Physics Requirements Document $1.4-001$.
} 
2. The electron trajectory must remain straight to $5 \mu \mathrm{m}$ over $10 \mathrm{~m}$. Within the undulator, the trajectory must be tuned to be straight to $2 \mu \mathrm{m}$.

3. The first field integral of $B_{x}$ and $B_{y}$ must be tuned to be below $40 \times 10^{-6} \mathrm{Tm}$. The second field integral of $B_{x}$ and $B_{y}$ must be tuned to be below $50 \times 10^{-6} \mathrm{Tm}^{2}$.

4. The electron motion must remain in phase with the radiation wave to better than 10 degrees through the undulator.

5. The phase advance of the radiation wave must be $113 \times 360^{\circ}$ over a distance of 3.656 $\mathrm{m}$ centered on the undulator.

6. The position of the line along which $K$ has the required value must be known relative to tooling balls on the undulator to $50 \mu \mathrm{m}$ at both ends of the undulator.

The pole cant angle will be used in setting $K$ to the required value. The undulator will be rough tuned along the magnetic center line. The undulator gap will be set with an accuracy of $10 \mu \mathrm{m}$ using shims under the pole assemblies. With a pole cant angle of 4.5 mrad, a horizontal undulator translation of up to $2.2 \mathrm{~mm}$ will be required to achieve the correct gap, and therefore the correct $K$ value. The undulator will be fine tuned on the translated line where the $K$ value is correct. The undulator will be fiducialized so that the beam axis is on the horizontally translated line where the $K$ value is correct.

In addition to the preceding tuning and fiducialization requirements, we impose the following operational requirements.

1. The reference undulator must be sent through the laboratory for measurements after every 4'th undulator. At this time, the alignment of the measurement benches must also be checked.

2. All girders must be measured to check for magnetic effects coming from both permanent magnetization and permeability differences which affect the Earth's magnetic field. The magnetic field above each girder at the beamline height must be the same as the girder used for the measurement bench to within 0.3 Gauss. This limit is set by the capabilities of the magnetic shield.

3. The magnetic field in the tunnel must be measured, and the magnetic field at the test stand must be measured. The fields must agree to within 0.3 Gauss. This limit is set by the capabilities of the magnetic shield.

\section{Measurement Laboratory}

The Magnetic Measurement Facility, MMF, has two test benches, a coarse tuning bench and a fine tuning bench. The coarse tuning bench will be used for setting the undulator gap so that the desired $K$ value can be reached along a line within $2.2 \mathrm{~mm}$ horizontally of the undulator magnetic center line. The undulator will be tuned with corrections in anticipation of a magnetic shield. Once the undulator is roughly tuned and access to bolts in the titanium housing is no longer required, a magnetic shield will be installed. At this 
point, the undulator is ready for fine tuning. The undulator will be moved to the fine tuning bench and re-aligned. The fine tuning bench will have a wide array of measurement devices allowing high precision measurements to get the undulator within specifications and fiducialized.

The equipment used at each bench is illustrated in figure 1. The coarse tuning bench
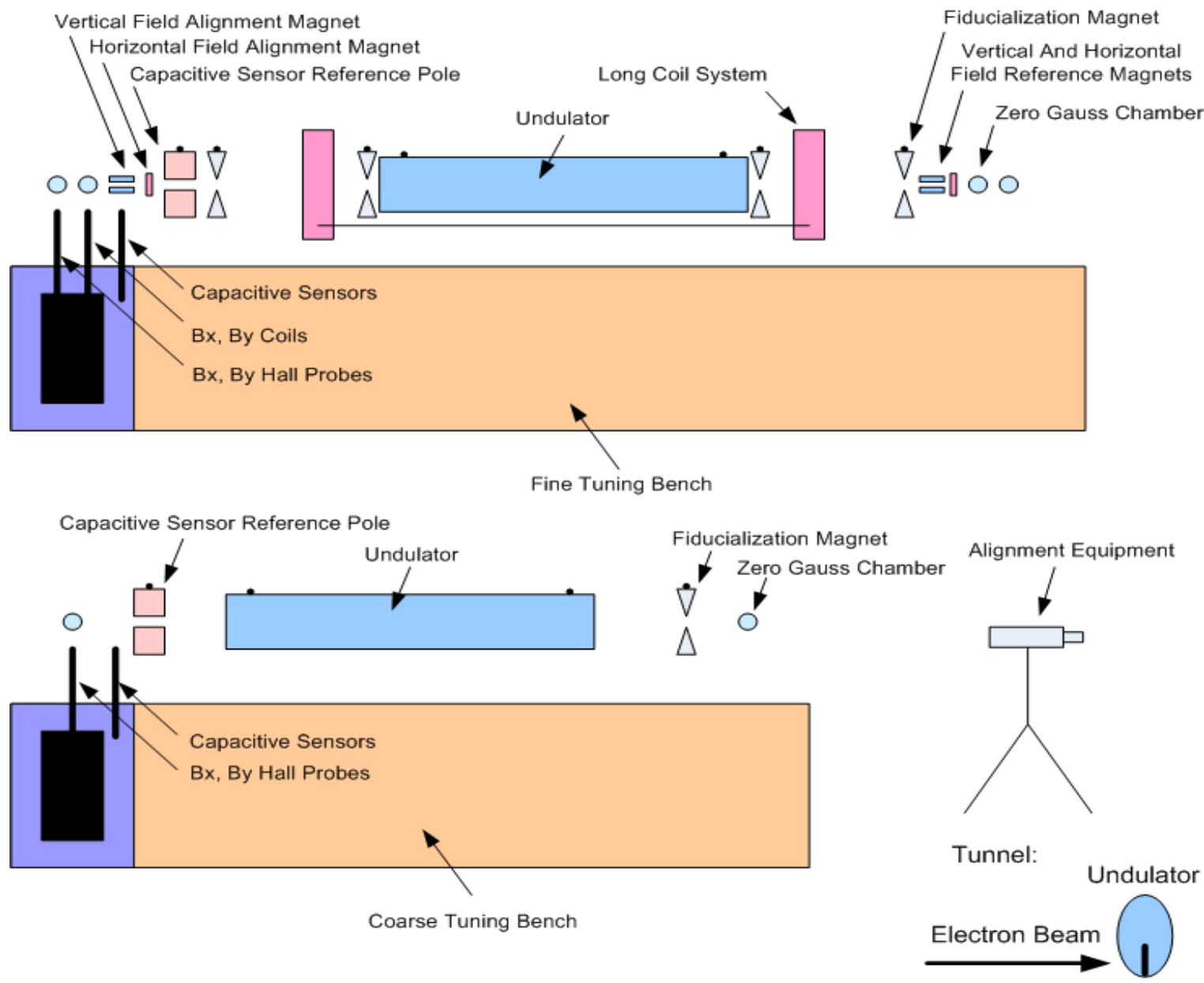

Figure 1: The MMF contains both a coarse tuning bench and a fine tuning bench.

will have a set of capacitive sensors to align the undulator to the bench. Hall probes will be used to measure $B_{x}$ and $B_{y}$ for the tuning.

The fine tuning bench will also use capacitive sensors to align the undulator to the bench. Hall probes will be used for most of the magnetic measurements. Short coils will complement the Hall probes for local field integral measurements. A long coil will be used for global field integral and field uniformity measurements. The undulator fiducialization will occur on this bench. Sets of fiducialization magnets and reference poles on stands at the ends of the undulator will be used as checks to avoid fiducialization errors. The vertical and horizontal field alignment magnets are used to set the probe angles. The vertical and 
horizontal reference magnets are used to check the Hall probe measurements.

Each magnetic measurement probe starts in a zero Gauss chamber and ends the measurement in a zero Gauss chamber. There is no field in these chambers, so any reading is due to offsets in the probe electronics. A linear fit is made to the beginning and end measurements in the zero Gauss chambers and the fit is subtracted from the measurements in the undulator as a zero offset correction.

\section{Rough Tuning Test Plan}

1. Preliminaries

Measure the earth's field at the test stand and calculate the correction field to add to the measured data to anticipate the magnetic shield.

2. Place the undulator

(a) Attach kinematic mounting feet.

(b) Put the undulator on the cam movers which are on top of the support stands. A steel girder and stands are not required for this part of the tuning.

(c) Attach thermistors.

3. Mechanically align the undulator to the test stand

(a) Have an alignment crew roughly place the undulator so the probes go through it without touching.

(b) Use capacitive sensors to measure the undulator position.

(c) Use the cam movers to move the undulator into position.

4. Magnetically align the Hall probe to the undulator

(a) Measure along the undulator with the Hall probe to find the pole positions.

(b) At every $N^{\prime}$ th pole $(N \simeq 15)$, move the Hall probe in $\mathrm{x}$ and $\mathrm{y}$ and find the magnetic center. Fit the magnetic field centers as a function of $\mathrm{z}$. Move the probe to the field center line.

(c) Correct pitch and yaw of the magnetic center line with the cam movers, if necessary, and repeat step (b).

(d) The magnetic center line defines $x=0, y=0$ for the Hall probe position.

5. Rough tuning

(a) Tune the $\mathrm{x}$ trajectory and the $\mathrm{y}$ trajectory.

(b) In step (a), include the correction to anticipate the magnetic shield.

6. Gap adjustment 
(a) Move the Hall probe to the magnetic center line.

(b) Measure K.

(c) Adjust the tapered shims so that $K$ has the required value for the undulator. A table of required $K$ value for each undulator position is given in the following section. If tapered shim motions of more than $10 \mathrm{~mm}$ are required to set $K$, adjust the gap by adding plain shims which act as spacers if the field is too weak, or replace the tapered shims with thinner ones if the field is too strong.

7. Check the alignment

(a) If the gap was changed, check the mechanical alignment of the undulator with the capacitive sensors. Adjust if necessary.

(b) If the gap was changed, check that the probe is still magnetically centered in the undulator. Adjust if necessary.

(c) Measure $K$ at $y=0, \pm 50 \mu \mathrm{m}$. Make sure the minimum $K$ occurs at $y=0$. Adjust if necessary.

8. Rough tuning (This step may not be necessary after one gets experience with the magnetic shield.)

(a) Tune the $\mathrm{x}$ trajectory, $\mathrm{y}$ trajectory, and the phase.

(b) In step (a), include the correction to anticipate the magnetic shield.

9. Magnetic shield

(a) Add the magnetic shield.

(b) Remove the correction in the data analysis used to anticipate the effect of the magnetic shield.

10. Rough tuning

Tune the $\mathrm{x}$ trajectory, y trajectory, and the phase.

\section{Required K Value For Each Undulator Position}

The $K$ value for each undulator must be set according to the beam energy at the undulator location. A table of the required $K$ values is given in figure 2 .

\section{Fine Tuning Test Plan}

1. Preliminaries

In an initial setup, measure the difference between the field integrals from the Hall probe and the short coils and calculate the correction field to add to the Hall probe data so that it agrees with the coil measurements. Add this correction to all Hall probe measurement data. 


\begin{tabular}{|r|c|c|}
\hline pos & $y$ & Keff \\
\hline 1 & 26692.70 & 3.500000 \\
\hline 2 & 26690.65 & 3.499557 \\
\hline 3 & 26688.61 & 3.499114 \\
\hline 4 & 26686.34 & 3.498623 \\
\hline 5 & 26684.30 & 3.498180 \\
\hline 6 & 26682.25 & 3.497737 \\
\hline 7 & 26679.99 & 3.497246 \\
\hline 8 & 26677.94 & 3.496803 \\
\hline 9 & 26675.90 & 3.496360 \\
\hline 10 & 26673.63 & 3.495869 \\
\hline 11 & 26671.59 & 3.495426 \\
\hline 12 & 26669.54 & 3.494984 \\
\hline 13 & 26667.27 & 3.494492 \\
\hline 14 & 26665.23 & 3.494049 \\
\hline 15 & 26663.19 & 3.493607 \\
\hline 16 & 26660.92 & 3.493115 \\
\hline 17 & 26658.88 & 3.492672 \\
\hline
\end{tabular}

\begin{tabular}{|r|c|c|}
\hline pos & $y$ & Keff \\
\hline 18 & 26656.83 & 3.492230 \\
\hline 19 & 26654.56 & 3.491738 \\
\hline 20 & 26652.52 & 3.491296 \\
\hline 21 & 26650.48 & 3.490853 \\
\hline 22 & 26648.21 & 3.490361 \\
\hline 23 & 26646.17 & 3.489919 \\
\hline 24 & 26644.12 & 3.489476 \\
\hline 25 & 26641.85 & 3.488984 \\
\hline 26 & 26639.81 & 3.488542 \\
\hline 27 & 26637.77 & 3.488099 \\
\hline 28 & 26635.50 & 3.487608 \\
\hline 29 & 26633.46 & 3.487165 \\
\hline 30 & 26631.41 & 3.486722 \\
\hline 31 & 26629.14 & 3.486231 \\
\hline 32 & 26627.10 & 3.485788 \\
\hline 33 & 26625.06 & 3.485345 \\
\hline
\end{tabular}

Figure 2: This table gives the $\mathrm{K}$ value for each undulator location. The $\mathrm{K}$ value must be set according to the electron beam energy at the location of the undulator. 
2. Place the undulator

Place the undulator on the cam movers on top of the test stand. A steel girder and stands are required for this part of the test.

3. Mechanically align the undulator to the test stand

(a) Have an alignment crew roughly place the undulator so the probes go through it without touching.

(b) Use capacitive sensors to measure the undulator position.

(c) Use the cam movers to move the undulator into position.

4. Magnetically align the Hall probe to the undulator

(a) Measure along the undulator with the Hall probe to find the pole positions.

(b) At every $N$ 'th pole $(N \simeq 15)$, move the Hall probe in $\mathrm{x}$ and $\mathrm{y}$ and find the magnetic center. Fit the magnetic field centers as a function of z. Move the probe to the field center line.

(c) Correct pitch and yaw of the magnetic center line with the cam movers, if necessary, and repeat step (b).

(d) The magnetic center line defines $x=0, y=0$ for the Hall probe position.

5. Determine the tuning axis

(a) Measure $K$ at $y=0, x=-2.5,-2.0, \ldots, 2.5 \mathrm{~mm}$.

(b) Fit the measurements to find the $x$ location where $K$ has the desired value.

(c) Translate the coordinate system. Redefine the $x=0$ position as the position found in step (b) where $K$ has the desired value.

(d) Measure $K$ at the new $x=0$ position as a check.

(e) Leave the Hall probe at $x=0, y=0$ and perform all tuning along the line at this position.

6. Rough tuning

Tune the $\mathrm{x}$ trajectory, $\mathrm{y}$ trajectory, and the phase.

7. Add phase matching shims

Correct the phase advance at the entrance and the exit.

8. Adjust the field integrals

(a) Measure the field integrals of both $B_{x}$ and $B_{y}$ with a long coil.

(b) Use trajectory shims at the entrance to null the second field integrals.

(c) Use trajectory shims at the exit to null the first field integrals. 
9. Measure the field uniformity

(a) With the Hall probe, measure the $\mathrm{x}$ trajectory, y trajectory, phase, and the $K$ value at $y=0, x=-4,0,4 \mathrm{~mm}$ and $x=0, y=-100,0,100 \mu \mathrm{m}$.

(b) Add quadrupole and sextupole shims as necessary to make these quantities uniform.

10. Final checks before gluing shims in place

(a) Check the $\mathrm{x}$ trajectory, $\mathrm{y}$ trajectory, and the phase. Correct if necessary.

(b) Check the first and second field integrals of $B_{x}$ and $B_{y}$. Correct if necessary.

11. Glue shims in place

Remove the y trajectory, quadrupole, sextupole, and phase shims from the gap. (This may be unnecessary for the phase shims.) Apply glue to the shims and replace.

12. Check the gap

(a) Visually inspect the gap to make sure no shim is protruding into the gap.

(b) Check the gap with a clearance gauge to make sure no shim is protruding into the gap.

(c) Fix problems as necessary.

13. Check for errors after gluing

(a) Check the $\mathrm{x}$ trajectory, $\mathrm{y}$ trajectory, the phase, and the first and second field integrals. Use the Hall probe and short coils.

(b) Repeat at $y=0, x=-4,0,4 \mathrm{~mm}, x=0, y=-100,0,100 \mu \mathrm{m}$.

(c) Fix problems as necessary.

14. Measure $B_{x}$ and $B_{y}$ in the retracted position

(a) Move the Hall probe back to the beam position when the undulator is retracted.

(b) Measure $B_{x}$ and $B_{y}$ and record the field values. No corrections are made.

15. Final results data set

(a) Perform measurements and calculate the $\mathrm{x}$ trajectory, $\mathrm{y}$ trajectory, phase, and the first and second field integrals of $B_{x}$ and $B_{y}$ at all the following locations: $x=-6,-5, \ldots, 6 \mathrm{~mm}, y=-0.2,-0.08,0,0.08,0.2 \mathrm{~mm}$. Place these $13 \times 5=65$ data sets in a special results folder.

(b) Use the long coil to measure the first and second field integrals of $B_{x}$ and $B_{y}$. Center the measurement on $x=-6,-3,0,3,6, y=0 \mathrm{~mm}$. Place this data in the tuning results folder. 
16. Find the required $\mathrm{K}$ using the canted poles

(a) Use the data from the previous step to determine $K$ vs $x$ at $y=0$.

(b) Fit the data to find the $x$ position where $K$ has the required value.

(c) Move the Hall probe to this $x$ position and perform a measurement to verify the $K$ value.

17. Add fiducialization magnets

(a) Add the fiducialization magnets to the undulator ends.

(b) Measure the offset from the line where $K$ has the required value to the center of the fiducialization magnets.

(c) Perform this measurement at both ends of the undulator.

18. Rough fiducialization

Perform a rough fiducialization using alignment equipment. This is meant only to limit any possible errors.

19. Measure roll

Using a level on the appropriate flats, measure the roll at each end of the undulator.

\section{Fiducialization on the CMM}

(a) Move the undulator to the CMM.

(b) Measure offsets from the tooling balls on the fiducialization magnets to the tooling balls on the undulator.

\section{Measurement Results}

All raw data and analysis results will be available from the SLAC web site. The data will be stored in a directory structure as show in figure 3. The top level directory is Magdata, followed by $L C L S$, followed by the magnet type Undulator. In the Undulator directory, there is a folder for each undulator named by the serial number. For each undulator, Dataset directories are made. When the undulator comes back for multiple measurements over time, each set of measurements goes into a new dataset. Within each dataset, the Temperature, Rough Tuning, Fine Tuning, Tuning Results, and Fiducialization folders are created. Each contains all the relevant measurements. When multiple runs are required, the data and analysis results from each run will go into a run folder. A list file will give a description of each run. All data files will be text files. There will be several types of data files with different formats depending on the measurement. Analysis results will be in both text files and postscript plot files. A brief description of the types of files encountered in the various runs follows.

Capacitive sensor data will include a file containing the $z$ position and all measured distances from the sensors. Another file will contain the calculated undulator position and orientation. 


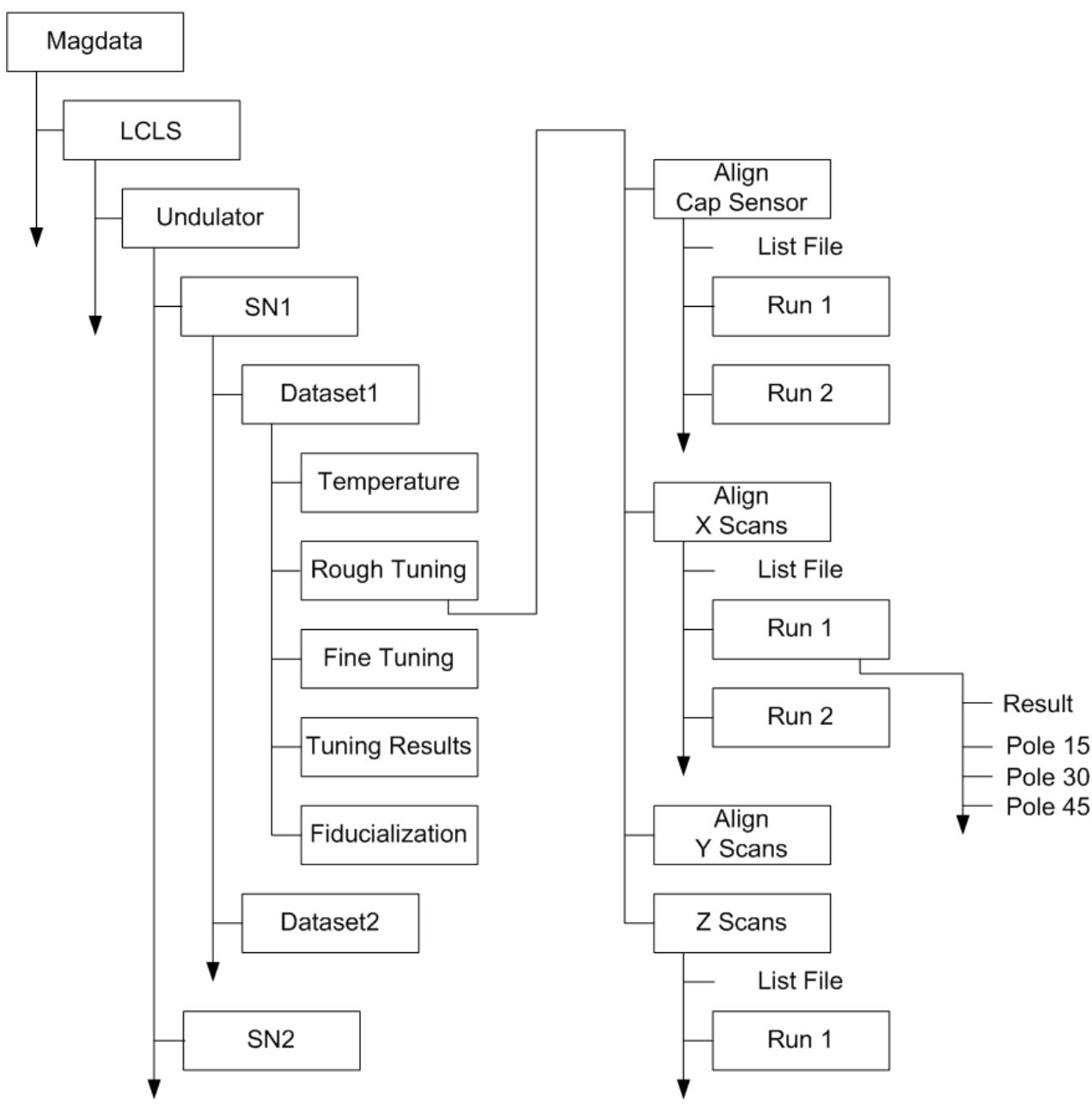

Figure 3: The undulator measurement data will be stored in a directory structure.

The rough tuning bench magnetic measurement data will consist of columns of the $z$ positions, the $V_{x}$ and $V_{y}$ Hall probe voltages, and $B_{x}$ and $B_{y}$ values. Parameters, such as the Hall probe calibration file, will be included in a header. Two separate SLAC analysis programs will be run on the data. The first computes the trajectories, phase, etc. The second computes the shims required to tune the undulator. These programs output text files containing the results and also postscript files containing plots.

The fine tuning bench magnetic measurement raw data will consist of columns of the $z$ positions, the $V_{x}$ and $V_{y}$ Hall probe voltages, and $B_{x}$ and $B_{y}$ values from the Hall probe. In addition, it will contain the $I V_{x}$ and $I V_{y}$ voltages from the integrator outputs of the coil voltages, and $B_{x}$ and $B_{y}$ values determined from the coils. The same two SLAC analysis programs will be run as for the rough tuning bench. The coil data will be compared to the Hall probe data.

The long coil data will consist of the $(x, y)$ location of the coil, parameters of the coil motion, the integrated voltage, and the first and second field integrals. Multiple 
measurements will be done. Each measurement will be recorded and the average and rms deviation will be provided. Output files will be text files containing a header and the first and second integrals of $B_{x}$ and $B_{y}$.

After the tuning runs are complete, a special set of final measurement runs is made. The analysis results from these runs will go into a final tuning results folder. It will contain the results from step 15, "Final results data set", in the fine tuning test plan. For each of the scans done at different $x$ and $y$ locations, a text file containing the results will be generated. The file will contain a header and calculated results such as the $K$ value, the phase change into the undulator, the phase change out of the undulator, and the phase change in the cell. It will also contain columns of data as follows

$$
z, B_{x}, B_{y}, I_{1 x}, I_{1 y}, I_{2 x}, I_{2 y} \text {, slippage, phase, } \ldots
$$

One line of data will be given for each measurement point, making the files around 30,000 lines long. The files are meant to be imported and used by other analysis programs. A Matlab data file will also be placed in the folder along with the text file. The Matlab file will contain an extensive set of quantities calculated in the analysis program. Additionally, the field measurements along the beam line when the undulator is in the retracted position will be placed in the results folder.

The fiducialization data will be put in its own folder. The data comes from several sources: scale readings from the measurement bench, offsets between the center of the fiducialization magnets and tooling balls, CMM data, and alignment crew data. All files will be text files containing a header and the measured values. A program will read the data and write a fiducialization report containing the final results. 